\title{
Effect of exchange bias on the electrical resistivity of Pd doped NiMn thin films: Two-channel Kondo system
}

\author{
Y. Öner ${ }^{\text {a) }}$ and O. Kamer \\ Department of Physics, Istanbul Technical University, TR-34469 Istanbul, Turkey \\ Joseph H. Ross, Jr. \\ Department of Physics, Texas A\&M University, College Station, Texas 77843-4242
}

(Received 9 May 2006; accepted 26 September 2006; published online 14 December 2006)

\begin{abstract}
Electrical resistivity measurements have been carried out for both flash-evaporated reentrant spin glasses (RSGs) $\left(\mathrm{Ni}_{76-x} \mathrm{Pd}_{x}\right) \mathrm{Mn}_{24}$ and $\mathrm{Ni}_{74.5} \mathrm{Mn}_{23.5} \mathrm{Pd}_{2}$, as well as $\mathrm{Ni}_{75} \mathrm{Mn}_{23} \mathrm{Pd}_{2}$, a pure SG. These measurements were carried out at temperatures down to $4 \mathrm{~K}$. We observed a very deep resistivity minimum at about $75 \mathrm{~K}$ for $\mathrm{Ni}_{74} \mathrm{Mn}_{24} \mathrm{Pd}_{2}$. It was found previously [Öner et al., J. Appl. Phys. 89, 7044 (2001)] that this sample shows the largest coercivity and exchange unidirectional anisotropy among these films. In addition, magnetization measurements show that this takes place just on the border of the RSG such that it could be handled as a superparamagnetic sample. Previously it was assumed that the exchange bias created in the sample between the domains plays the dominant role in the resistivity minimum. On the other hand, in order to account for the temperature dependence of the resistivity below the minimum we have analyzed these data using the Kondo, two-channel Kondo, weak localization, and Cochrane models for structural disorder based on the Anderson mechanism. We have deduced that the two-channel Kondo model gives the best agreement with the data; a logarithmic temperature dependence $\Delta \rho(T)=\beta \log _{10}\left(T / T_{K}\right)$, was observed at the temperatures below $T_{f}$ accompanied by a resistivity behavior $\Delta \rho(T)=\rho_{0 m}(0)\left(1-\alpha T^{1 / 2}\right)$, at lower temperatures. All parameters deduced from the fitting correlate consistently with the strength of the exchange anisotropy and coercivity in the RSG films, and thus provide a separate measure of the presence of antiferromagnetically coupled domains in these materials. (c) 2006 American Institute of Physics. [DOI: 10.1063/1.2400094]
\end{abstract}

\section{INTRODUCTION}

Disordered $\mathrm{Ni}_{1-x} \mathrm{Mn}_{x}$ alloys have very peculiar and interesting electrical and magnetic behaviors with a strong dependence on the composition and the magnetic and thermal histories. Near a multicritical point at a composition $x$ $=0.24$, reentrant spin-glass $(\mathrm{RSG})$ behavior is observed, ${ }^{1}$ in which a ferromagnetically ordered state exhibits a spontaneous transformation to a disordered magnetic state upon decreasing temperature. It is now generally agreed that this transformation involves canting of antiferromagnetically coupled spins, ${ }^{2-4}$ although the ferromagnetic state itself is not a fully ordered one, ${ }^{5}$ but includes reversed domains. The electrical resistivity is an important means to characterize magnetic disorder and low-lying excitation in such materials. It has been shown ${ }^{6,7}$ that NiMn and Pt doped NiMn alloys exhibit resistivity minima at low temperatures, with the resistivity increase reflecting the emergence of a more highly disordered magnetic phase. In addition, Sato et al. ${ }^{8}$ have pointed out that the resistivity minimum temperature $\left(T_{m}\right)$ correlates with the spin-glass transition temperature $T_{f}$. However, for Mn concentrations smaller than the $x=0.24$ multicritical point the minimum shifts to lower temperature with increasing thickness, whereas this minimum shifts to higher

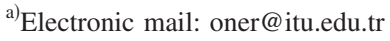

temperature for Mn concentrations above the multicritical concentration. It appears that the spin correlations have different characters in these two regimes.

One of the most important contributions to the resistivity upturn in disordered magnetic systems at low temperatures arises from electron scattering by localized spins and is magnetic in origin. However, a number of other contributions can arise, ${ }^{9}$ including the magnetic single-ion Kondo scattering and a model associated with quantum tunneling between metastable two-level states developed by Cochrane et al. ${ }^{10}$ These tunneling states can be associated with structural as well as magnetic disorder, and indeed a low-temperature resistivity minimum is commonly observed in nonmagnetic metallic glasses. ${ }^{11}$

Previously, it was shown ${ }^{7,12}$ that amorphous NiMn films exhibit a RSG behavior similar to that of the corresponding polycrystalline materials. Recently, we have carried out magnetic measurements of flash-evaporated Pd doped NiMn alloys to investigate the role of $\mathrm{Pd}$ atoms on the magnetic states of these alloys for zero field cooled (ZFC) or FC cases. ${ }^{13}$ The irreversibility behavior observed in this system was explained in terms of the antiferromagnetic alignment of magnetic domains in the ferromagnetic state. Here we report results of resistivity measurements on these thin films, in order (i) to bring out the role of Pd impurities on the anisotropies, which are strongly sensitive to local magnetic order, (ii) to provide a better understanding of the resistivity minimum in this system, and (iii) to study the correlation 

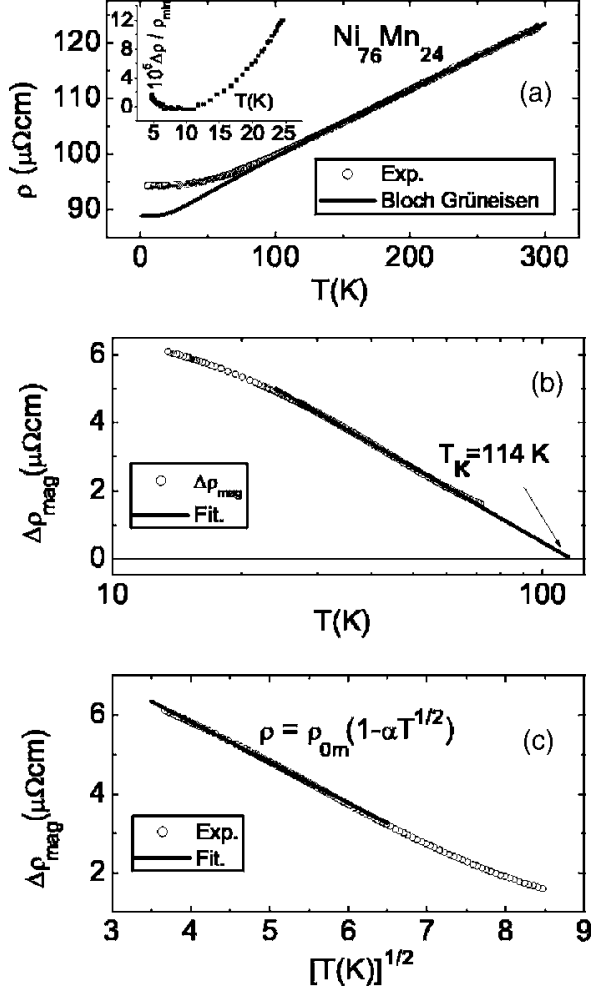

FIG. 1. (a) Electrical resistivity as a function of temperature for $\mathrm{Ni}_{76} \mathrm{Mn}_{24}$. Inset shows the normalized resistivity with respect to the resistivity value at $T_{m}$, where the resistivity passes through a minimum, as a function of temperature, plotted on an expanded scale. Solid line shows the fit to the BlochGrüneisen expression at higher temperatures. (b) The magnetic contribution to the resistivity $\Delta \rho_{\operatorname{mag}}$ plotted vs $\log _{10} T$. The solid line represents Eq. (2) with values of $\beta$ and $T_{K}$ given in Table II. The Kondo temperature $T_{K}$ was determined from the intersection of the logarithmic resistivity line with the temperature axis. Note also the obvious deviation from the linear behavior of resistivity with respect to $\log T$ at lower temperatures. (c) $\Delta \rho_{\text {mag }}$ plotted vs $T^{0.5}$. The solid line represents the expression indicated in the figure [Eq. (3)]. Values of the fitting parameters $\alpha$ and $\rho_{0 m}$ are given in Table II.

between the low-temperature resistivity and the strength of the exchange anisotropy and coercivity in these films.

\section{EXPERIMENT}

Films of compositions of $\left(\mathrm{Ni}_{76-x} \mathrm{Pd}_{x}\right) \mathrm{Mn}_{24}$ ( $x=0,2$, and 5), $\mathrm{Ni}_{74.5} \mathrm{Mn}_{23.5} \mathrm{Pd}_{2}$, and $\mathrm{Ni}_{75} \mathrm{Mn}_{23} \mathrm{Pd}_{2}$ were prepared using a flash evaporation technique. These were identical films to those described in the earlier magnetic study. ${ }^{13} \mathrm{X}$-ray spectra taken on these samples showed no Bragg peaks but were characterized by a broad structureless intensity with a maximum at low angles that is a characteristic of the amorphous state. Atomic force microscopy (AFM) was used to control the quality of the sample. All these measurements provided clear evidence that they have a highly disordered, noncrystalline structure. The resistivity for these samples was measured using a standard dc four-probe setup over a $4-300 \mathrm{~K}$ temperature range. A calibrated Ge thermometer (GR-200A2500) was used to measure the temperature below $80 \mathrm{~K}$. For higher temperatures, a calibrated Pt thermometer was used.

\section{RESULTS}

Figures 1-5 show the temperature dependence of the electrical resistivity for $\mathrm{Ni}_{76} \mathrm{Mn}_{24}, \mathrm{Ni}_{74.5} \mathrm{Mn}_{23.5} \mathrm{Pd}_{2}$,
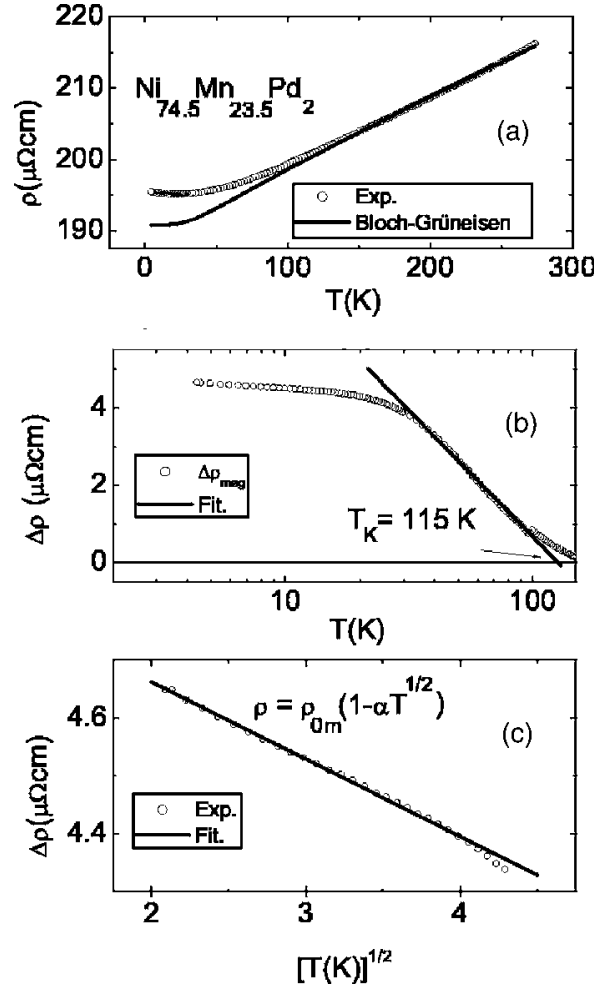

FIG. 2. (a) Electrical resistivity as a function of temperature for $\mathrm{Ni}_{74.5} \mathrm{Mn}_{23.5} \mathrm{Pd}_{2}$. Solid line shows the fit to the Bloch-Grüneisen expression at higher temperatures. (b) The magnetic contribution to the resistivity $\Delta \rho_{\text {mag }}$ plotted vs $\log _{10} T$. The solid line represents Eq. (2) with values of $\beta$ and $T_{K}$ given in Table II. (c) $\Delta \rho_{\text {mag }}$ plotted vs $T^{0.5}$. The solid line represents the expression indicated in the figure [Eq. (3)]. Values of the fitting parameters $\alpha$ and $\rho_{0 m}$ are given in Table II.

$\mathrm{Ni}_{74} \mathrm{Mn}_{24} \mathrm{Pd}_{2}, \mathrm{Ni}_{75} \mathrm{Mn}_{23} \mathrm{Pd}_{2}$, and $\mathrm{Ni}_{71} \mathrm{Mn}_{24} \mathrm{Pd}_{5}$, respectively. All resistivity curves show a similar temperature dependence behavior. The resistivity first decreases almost linearly with decreasing temperature and then changes its slope gradually. As the temperature is further decreased, the resistivity passes through a minimum and then increases. It should be noted that for $\mathrm{Ni}_{74.5} \mathrm{Mn}_{23.5} \mathrm{Pd}_{2}$ and $\mathrm{Ni}_{74} \mathrm{Mn}_{24} \mathrm{Pd}_{2}$, deep resistivity minima at about 23 and $75 \mathrm{~K}$, respectively, were observed. The $\mathrm{Ni}_{76} \mathrm{Mn}_{24}$ and $\mathrm{Ni}_{75} \mathrm{Mn}_{23} \mathrm{Pd}_{2}$ samples also exhibit shallow minima at about 8 and $10 \mathrm{~K}$, respectively [see the insets to Figs. 1(a) and 4(a)]. For the sample of $\mathrm{Ni}_{71} \mathrm{Mn}_{24} \mathrm{Pd}_{5}$, the resistivity minimum takes place at a temperature below our available lowest temperature, $4 \mathrm{~K}$. The values of the room temperature resistivity, the resistivity minimum temperatures, and the thicknesses are given in Table I, along with magnetic properties from Ref. 13 for comparison.

At higher temperatures, the resistivity is well described by the Bloch-Grüneisen equation ${ }^{14}$ expressed as follows:

$$
\rho_{s}=\rho_{\text {os }}+4 R T\left(T / \Theta_{D}\right)^{4} J_{5}\left(\Theta_{D} / T\right),
$$

where $\Theta_{D}$ is the Debye temperature, $J_{5}\left(\Theta_{D} / T\right)$ is the Grüneisen function, $R$ refers to the linear dependence at high temperature, and $\rho_{\mathrm{os}}$ is the residual resistivity. The BlochGrüneisen theory can apply just as well for the distribution of phonons in amorphous and nanocrystalline materials as in crystalline ones, ${ }^{15,16}$ as long as the electron mean free path is at least several near-neighbor distances. The resistivities observed here are in the metallic range, and small enough to 

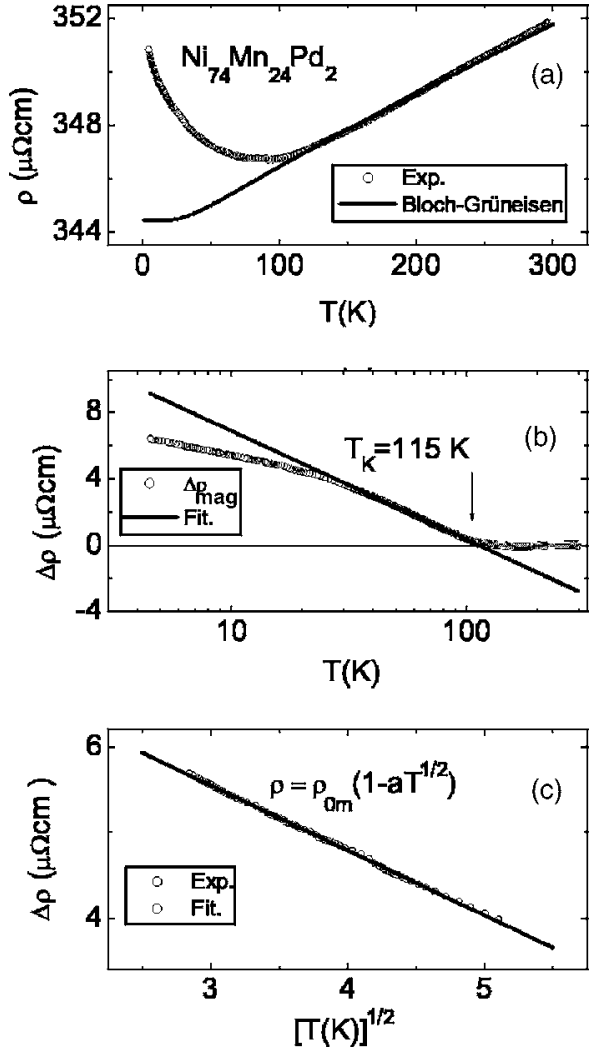

FIG. 3. (a) Electrical resistivity as a function of temperature for $\mathrm{Ni}_{74} \mathrm{Mn}_{24} \mathrm{Pd}_{2}$. Solid line shows the fit to the Bloch-Grüneisen expression at higher temperatures. (b) The magnetic contribution to the resistivity $\Delta \rho_{\text {mag }}$ plotted vs $\log _{10} T$. The solid line represents Eq. (2) with values of $\beta$ and $T_{K}$ given in Table II. (c) $\Delta \rho_{\text {mag }}$ plotted vs $T^{0.5}$. The solid line represents the expression indicated in the figure [Eq. (3)]. Values of the fitting parameters $\alpha$ and $\rho_{0 m}$ are given in Table II.

satisfy the latter condition. There is also a term in the resistivity of amorphous films due to scattering from vibrating randomly positioned atoms, which gives a $T^{2}$ temperature dependence. This term can dominate in metal-metalloid films such as Ni-P, ${ }^{16}$ however, in Ti-Co films this term is found to be small, ${ }^{15}$ and one would expect a similar situation to hold in $\mathrm{Ni}-\mathrm{Mn}$ films, as indeed was found from our fitting procedure.

The fitting parameters $\rho_{\mathrm{os}}$ and $\Theta_{D}$ obtained by applying Eq. (1) for each sample are listed in Table II. We note that the values of $\Theta_{D}$ vary between 180 and $220 \mathrm{~K}$, which are very close to those obtained for some transition metal based metallic glasses. ${ }^{17} \rho_{\mathrm{os}}$ is assumed due to a static structural disorder, of nonmagnetic origin, and falls between 80 and $350 \mu \Omega \mathrm{cm}$. The Bloch-Grüneisen curves (the continuous curves) are also shown in the top panes of Figs. 1-5.

The excess resistivity $\Delta \rho_{\mathrm{mag}}=\rho_{\text {alloy }}(T)-\rho_{s}(T)$ of these alloys is plotted versus $\log _{10} T$ up to $120 \mathrm{~K}$ for each sample (middle panes, Figs. 1-5). This remainder can be considered as due to magnetic contributions to the resistivity. These figures reveal that the magnetic part of the resistivity for all samples begins to diverge upward with decreasing temperature and shows signs of saturation though it is still increasing even at our lowest available temperature of $4 \mathrm{~K}$. The full lines in the figures represent the following function:
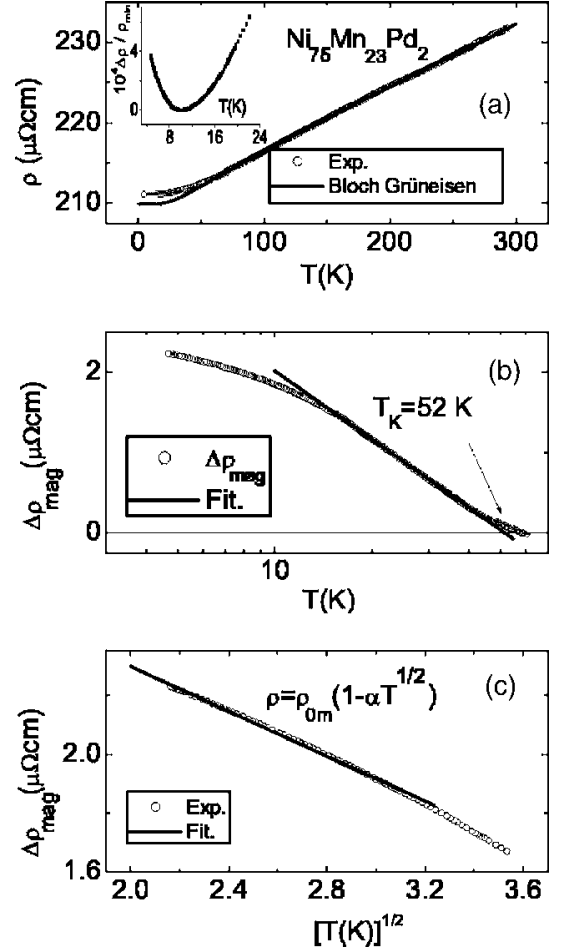

FIG. 4. (a) Electrical resistivity as a function of temperature for $\mathrm{Ni}_{75} \mathrm{Mn}_{23} \mathrm{Pd}_{2}$. The inset shows the normalized resistivity with respect to the resistivity value at $T_{m}$, where the resistivity passes through a minimum, as a function of temperature, on an expanded vertical scale. Continuous line shows the fit to the Bloch-Grüneisen expression at higher temperatures. (b) The magnetic contribution to the resistivity $\Delta \rho_{\operatorname{mag}}$ plotted vs $\log _{10} T$. The solid line represents Eq. (2) with values of $\beta$ and $T_{K}$ given in Table II. (c) $\Delta \rho_{\text {mag }}$ plotted vs $T^{0.5}$. The solid line represents the expression indicated in the figure [Eq. (3)]. Values of the fitting parameters $\alpha$ and $\rho_{0 m}$ are given in Table II.

$$
\Delta \rho(T)=\beta \log _{10} \frac{T}{T_{K}},
$$

where the values of the fitting parameters $\beta$ and $T_{K}$ together with the fitting ranges are presented in Table II. As indicated in the middle panes of Figs. 1-5, this logarithmic behavior provides a good fit to the results down to $40 \mathrm{~K}$ for $\mathrm{Ni}_{76} \mathrm{Mn}_{24}$, $\mathrm{Ni}_{74.5} \mathrm{Mn}_{23.5} \mathrm{Pd}_{2}$, and $\mathrm{Ni}_{74} \mathrm{Mn}_{24} \mathrm{Pd}_{2}$ and down to $10 \mathrm{~K}$ for $\mathrm{Ni}_{71} \mathrm{Mn}_{24} \mathrm{Pd}_{5}$ and $\mathrm{Ni}_{75} \mathrm{Mn}_{23} \mathrm{Pd}_{2}$. However, below these temperatures the experimental data fall below the logarithmic lines. At lower temperatures the expression

$$
\rho=\rho_{0 m}\left(1-\alpha T^{1 / 2}\right)
$$

provides a good description of the resistivities of all samples. To demonstrate this, figures 1(c), 2(c), 3(c), 4(c), and 5(c) show the lower-temperature excess resistivity $\Delta \rho_{\text {mag }}$, plotted versus $T^{1 / 2}$, along with fitting curves (the continuous curves) obtained by using Eq. (3). Values of the coefficients $\rho_{0 m}$ and $\alpha$ together with the fitting ranges of temperature are presented in Table II.

From our previous magnetization data (Ref. 13), we found that the first three samples given above are RSGs, whereas the $\mathrm{Ni}_{75} \mathrm{Mn}_{23} \mathrm{Pd}_{2}$ sample is a typical pure spin glass. The freezing temperatures, the coercivity values, and also the exchange field values (a shift along the field axis of the magnetization hysteresis loop) have been taken from Ref. 13 and 

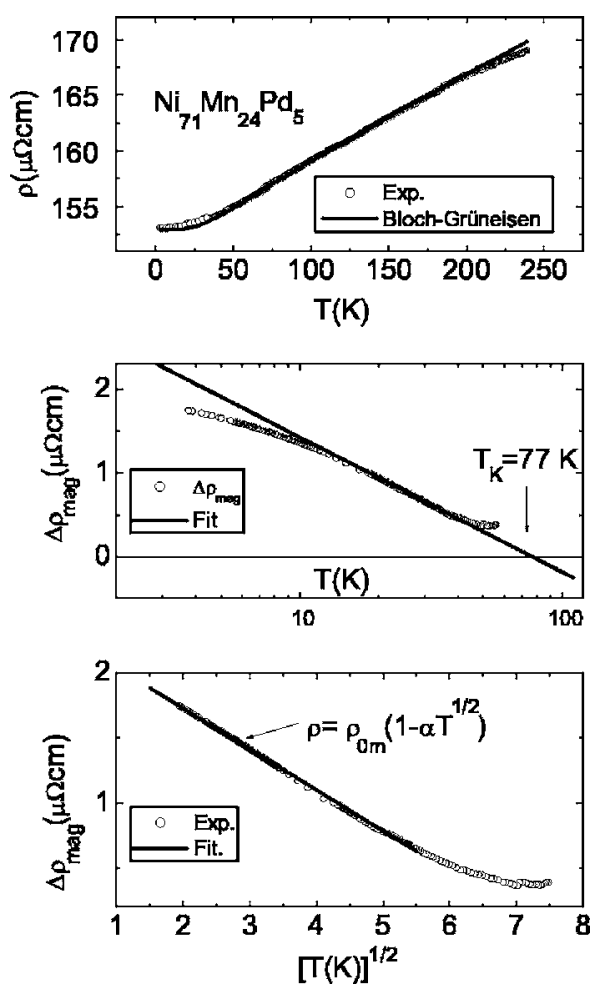

FIG. 5. (a) Electrical resistivity as a function of temperature for $\mathrm{Ni}_{71} \mathrm{Mn}_{24} \mathrm{Pd}_{5}$. Solid line shows the fit to the Bloch-Grüneisen expression at higher temperatures. (b) The magnetic contribution to the resistivity $\Delta \rho_{\mathrm{mag}}$ plotted vs $\log _{10} T$. The solid line represents Eq. (2) with values of $\beta$ and $T_{K}$ given in Table II. (c) $\Delta \rho_{\text {mag }}$ plotted vs $T^{0.5}$. The solid line represents the expression indicated in the figure [Eq. (3)]. Values of the fitting parameters $\alpha$ and $\rho_{0 m}$ are given in Table II.

summarized in Table I. Note that the depth of the resistivity minimum increases with increasing coercivity and exchange unidirectional anisotropy. Indeed, it can be seen from Fig. 3 that the $\mathrm{Ni}_{74} \mathrm{Mn}_{24} \mathrm{Pd}_{2}$ sample having the largest coercivity and exchange anisotropy among these samples gives a much deeper resistivity minimum at higher temperature. It is also interesting to note that this sample takes place just on the border of the RSG such that it could be handled as a superparamagnetic sample.

\section{ANALYSIS AND DISCUSSION}

We will first discuss the temperature dependence of the term $\Delta \rho_{\text {mag }}$, which we ascribe to the magnetic part of the resistivity. As indicated in Figs. 1-5, the rapid increase in the excess resistivity at higher temperatures levels off at lower temperatures. One possibility for this behavior is the singleion Kondo effect: For ferromagnetic glassy metals, though the exchange interaction might be expected to remove any magnetic degeneracy, the random magnetic disorder may leave some spins unquenched thus allowing a Kondo mechanism to persist. ${ }^{5}$ In this case a characteristic logarithmic behavior is expected for the resistivity. However, our attempts to fit to a resistivity term proportional to $-\ln T$ gave rather poor results. Other possible mechanisms responsible for an upturn in $\rho(T)$ near zero temperature include, for example, a linear increase in $T$ due to the screening of electron-electron interactions due to their slow diffusion in the disordered structure (Anderson weak localization). ${ }^{18}$ However, a linear fit does not fit our data satisfactorily, either. On the other hand, Cochrane et al. ${ }^{10}$ have also proposed a mechanism of structural origin, due to scattering by two-level systems. They obtained an analytic expression of the following form:

$$
\rho=\rho_{0}-A \ln \left(T^{2}+\Delta^{2}\right),
$$

where $\Delta$ is the energy difference between the two atomic tunneling states and $A$ is a constant depending on the number of contributing sites and the strength of the Coulomb interaction. We have used Eq. (4), with the addition of a temperature-independent term, $\rho_{0}$ for fitting the experimental data, and obtained a quite satisfactory fit (see Fig. 6). The values of the best fitting parameters $\rho_{0}, A$, and $\Delta$ are indicated in Fig. 6. The values of $\Delta$ for the other samples are also given in Table II. At first sight, one can ascribe these minima to the instability of the structural atomic order. However, similar resistivity minima have been observed for polycrystalline NiMn and Pt doped NiMn systems. ${ }^{19}$ It should be also be noted that fitted $\Delta$ values are much greater than $1 \mathrm{~K}$, which is an upper limit for samples whose resistivities are on the order of a few hundred $\mu \Omega \mathrm{cm}^{10}$

Close inspection of the parameters given in Tables I and II shows that the resistivity fitting parameters associated with the temperature-dependent behavior correlate with the coercivities (to be taken as a measure of the irreversibility) and the exchange anisotropies. Our previous transport measurements and this study lead us to conclude that resistivity minima observed in NiMn systems either in the polycrystalline phase or in the amorphous phase are of magnetic origin. We can rule out a mechanism of structural origin to account for the resistivity upturn below the freezing temperature. In order to interpret the temperature behavior at low temperatures and also clarify the relationship between the resistivity and the magnetic state of these systems, we suggest the fol-

TABLE I. Properties of samples used in this study: $\rho_{300}$, resistivity at $300 \mathrm{~K} ; T_{\min }$, temperature of resistivity minimum; $T_{f}$, magnetic freezing temperature; $\Delta H_{C}$, coercivity; and $H_{E}$, exchange unidirectional anisotropy.

\begin{tabular}{lcccccr}
\hline \hline \multicolumn{1}{c}{ Sample } & $\begin{array}{c}\text { Thickness } \\
(\mathrm{nm})\end{array}$ & $\begin{array}{c}\rho_{300} \\
(\mu \Omega \mathrm{cm})\end{array}$ & $\begin{array}{c}T_{\text {min }} \\
(\mathrm{K})\end{array}$ & $\begin{array}{c}T_{f} \\
(\mathrm{~K})\end{array}$ & $\begin{array}{c}\Delta H_{C} \\
(\mathrm{Oe})\end{array}$ & $\begin{array}{c}H_{E} \\
(\mathrm{Oe})\end{array}$ \\
\hline $\mathrm{Ni}_{76} \mathrm{Mn}_{24}$ & 105 & 124 & $\sim 8$ & $35^{\mathrm{a}}$ & $460^{\mathrm{a}}$ & $80^{\mathrm{a}}$ \\
$\mathrm{Ni}_{74.5} \mathrm{Mn}_{23.5} \mathrm{Pd}_{2}$ & 88 & 216 & 23 & $\cdots$ & $\cdots$ & $\cdots$ \\
$\mathrm{Ni}_{74} \mathrm{Mn}_{24} \mathrm{Pd}_{2}$ & 73 & 352 & 80 & $60^{\mathrm{a}}$ & $1450^{\mathrm{a}}$ & $100^{\mathrm{a}}$ \\
$\mathrm{Ni}_{75} \mathrm{Mn}_{23} \mathrm{Pd}_{2}$ & 58 & 232 & 10 & $50^{\mathrm{a}}$ & $280^{\mathrm{a}}$ & $5^{\mathrm{a}}$ \\
$\mathrm{Ni}_{71} \mathrm{Mn}_{24} \mathrm{Pd}_{5}$ & 100 & 178 & $<4$ & $50^{\mathrm{a}}$ & $560^{\mathrm{a}}$ & $30^{\mathrm{a}}$ \\
\hline \hline
\end{tabular}

${ }^{\mathrm{a}}$ From Ref. 13; measured at $T=3 \mathrm{~K}$. 
TABLE II. Parameters extracted from resistivity fits: $\theta_{D}$ is the Debye temperature; $R$ is a scaling parameter; $\rho_{0 s}$ is the residual resistivity from the Bloch-Grüneisen fit; $\rho_{0 m}$ and $\alpha$ are parameters from the $T^{1 / 2}$ fit [Eq. (3)]; $T_{K}$ is the Kondo temperature and $\beta$ is the multiplier for the Kondo fit; and $\Delta$ is the fitting parameter from the Cochrane fit.

\begin{tabular}{lcccccccc}
\hline \hline \multicolumn{1}{c}{ Sample } & $\begin{array}{c}\rho_{0 \mathrm{~s}} \\
(\mu \Omega \mathrm{cm})\end{array}$ & $\begin{array}{c}\Theta_{D} \\
(\mathrm{~K})\end{array}$ & $\begin{array}{c}R \\
(\mu \Omega \mathrm{cm} \mathrm{K})\end{array}$ & $\begin{array}{c}\rho_{0 \mathrm{~m}} \\
(\mu \Omega \mathrm{cm})\end{array}$ & $\begin{array}{c}\alpha^{\mathrm{a}} \\
\left(\mu \Omega \mathrm{cm} \mathrm{K}^{-1 / 2}\right)\end{array}$ & $\begin{array}{c}\beta^{\mathrm{a}} \\
(\mu \Omega \mathrm{cm})\end{array}$ & $\begin{array}{c}T_{K} \\
(\mathrm{~K})\end{array}$ & $\begin{array}{c}\Delta \\
(\mathrm{K})\end{array}$ \\
\hline $\mathrm{Ni}_{76} \mathrm{Mn}_{24}$ & 88 & 182 & 0.505 & 10 & $0.103(4-40 \mathrm{~K})$ & $7.33(40-70 \mathrm{~K})$ & 114 & 8.65 \\
$\mathrm{Ni}_{74.5} \mathrm{Mn}_{23.5} \mathrm{Pd}_{2}$ & 190 & 189 & 0.377 & 5 & $0.028(4-18 \mathrm{~K})$ & $6.51(40-120 \mathrm{~K})$ & 115 & 7.14 \\
$\mathrm{Ni}_{74} \mathrm{Mn}_{24} \mathrm{Pd}_{2}$ & 344 & 204 & 0.223 & 8 & $0.097(4-20 \mathrm{~K})$ & $6.55(40-120 \mathrm{~K})$ & 115 & 8.44 \\
$\mathrm{Ni}_{75} \mathrm{Mn}_{23} \mathrm{Pd}_{2}$ & 209 & 160 & 0.303 & 3 & $0.124(4-10 \mathrm{~K})$ & $2.61(15-40 \mathrm{~K})$ & 52 & 7.52 \\
$\mathrm{Ni}_{71} \mathrm{Mn}_{24} \mathrm{Pd}_{5}$ & 152 & 176 & 0.291 & 2 & $0.133(4-30 \mathrm{~K})$ & $1.61(10-40 \mathrm{~K})$ & 77 & 4.73 \\
\hline
\end{tabular}

${ }^{\mathrm{a}}$ The temperature range in parentheses shows the fitting range.

lowing model to be a possible mechanism, which is responsible for the upturn resistivity observed for the NiMn system at low temperatures.

As is well known, for metallic spin glasses there are many alternative spin configurations, separated by lowenergy barriers for local spin groups. This leads to a finite number of low-energy excitations. ${ }^{20}$ We believe that the existence of this low-energy spectrum, which is magnetic in origin, may provide a scattering channel for the electrons, analogous to the degree of freedom provided by a local spin in a Kondo system. The tunneling rate between local minima decreases with temperature. As the temperature is further decreased, the spins are frozen into one of the two potential minima. The interactions between resonating spins and magnetic two-level systems, of exchange origin, become dominant in the transport properties of the sample. Korenblit et $a l^{21}$ interpreted the results of inelastic neutron scattering on amorphous $\left(\mathrm{Fe}_{1-x} \mathrm{Mn}_{x}\right)_{75} \mathrm{P}_{16} \mathrm{~B}_{6} \mathrm{Al}_{3}$ spin-glass samples due to Aeppli et al. $^{22}$ in terms of magnetic two-level systems and claimed that the experimental results were a convincing evidence for two-level systems in amorphous Heisenberg ferromagnets with antiferromagnetic bonds. A similar model had already been suggested by Continentino ${ }^{23}$ for the tunneling rate from one metastable minimum to another via an interaction with spin waves and magnetic two-level systems (MTLSs) in a ferromagnet with frustration. We have used this model in our previous electron spin resonance (ESR) work done on amorphous NiMn and Pt doped NiMn thin

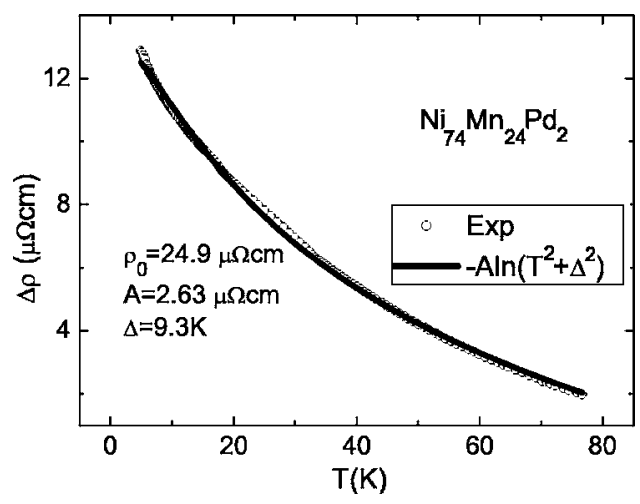

FIG. 6. Resistivity difference data $\Delta \rho_{\text {mag }}$ for $\mathrm{Ni}_{74} \mathrm{Mn}_{24} \mathrm{Pd}_{2}$, fitted to Eq. (4) as given in the text. The fitting parameters are also indicated in the figure. Note that the calculated values (solid line) for $\rho$ give a slight deviation from the experimental data at lower temperatures. films ${ }^{24}$ and compared our results with the prediction of this model. The resonance linewidths for these samples were satisfactorily interpreted in terms of the coupling between the resonating spins and magnetic two-level systems, considering that MTLSs are quasidegenerate and barrier heights $V$ are distributed according to $(1 / V) \exp \left(-V / V_{0}\right)$ and the relaxation time as $\tau_{0} \exp (V / k T)$. It appears that the scattering of resonating spins from MTLS induces tunneling (at a rate estimated to be a few times $10 \mathrm{GHz}$ from $X$-band ESR measurements), giving rise to an additional contribution to the lowtemperature resistivity. Another support for the existence of MTLS in amorphous spin glasses comes from the thermal conductivity $^{25}$ and magnetization studies ${ }^{26}$ done on some amorphous spin glasses $(\mathrm{PdCuSi})_{90} T M_{10}, T M=\mathrm{Mn}, \mathrm{Fe}$, and Co. In these works the authors have interpreted adequately their experimental results in terms of thermal activated MTLSs by inelastic scattering of phonons via spin-orbit interactions. Recently, Cox and co-workers ${ }^{27}$ have carried out a systematic renormalization-group treatment to reveal the physical properties of some thermodynamic quantities involving low-temperature logarithmic singularities for any disordered system. They have pointed out that the rate of electronic scattering from TLSs contributes to the resistivity, for which the temperature behavior depends on the ratio $V / T_{K}$, where $V$ is the energy difference between the two tunneling states and $T_{K}$ is the Kondo temperature, which can be estimated from the intersection of the logarithmic resistivity line with the temperature axis. If $V \ll T_{K}$, a region of $(\ln T)$ temperature behavior is followed by the region with $\rho / \rho_{0 m}=1-\alpha T^{1 / 2}$ temperature behavior. ${ }^{28,29}$ According to this model, a non-Fermi-liquid ( $\mathrm{nFL}$ ) ground state can be developed below $T_{K}$, while above $T_{K}$ a logarithmic temperature dependence of the electrical resistivity should be observed. In this model, the $\ln (T)$ singularity has its origin in the nonmagnetic interaction of itinerant electrons with the defect centers. In fact, this situation is mathematically analogous to the Kondo problem where the impurity is spin compensated by the conduction electrons. Hence, in this model the TLS corresponds to a Kondo spin-1/2 impurity, and the energy separation $V$ of the two TLS minima has its analogy in the Zeeman splitting of the Kondo impurity, while the partial waves (orbital degrees of freedom) of the conduction electrons interacting with the TLS correspond to the channel index in the classical Kondo problem. However, very recently, Aleiner et $a l^{30}$ have shown that two channel Kondo (with 
the orbital index of the particle) behavior can never be observed in the weak-coupling regime. But, in the strong coupling regime, Zaránd ${ }^{31}$ has suggested that the magnetic Kondo resonance would provide the resonance needed for the orbital Kondo effect. In this scenario, the magnetic correlations increase the coupling of the tunneling system and generate an orbital Kondo effect. In our study, the situation is different in that spin-dependent electron scattering causes some local groups of spins to change orientations with respect to the anisotropy field directions (such as triad anisotropy axes ${ }^{32}$ ). Here, the energy difference between two local minima of spin configurations corresponds to spin splitting. It should be noted that $T_{K}$ in our samples occurs in the temperature range of 30-80 K [above the temperature where the $\ln (T)$ behavior appears]. The thermal energy is enough to create a spectrum of magnons in domains (or within local groups of spins). As the temperature is further decreased, since the mean free path is so short for these samples (a few nanometers) only short-wavelength spin waves will cause scattering, and these have energies that are too high to be excited at lower temperatures. This means that the system stays in its lowest level at lower temperatures for a time which is long compared to the inverse tunneling rate of the electrons.

As for the temperature-independent term, there are two possible cases. Scattering from magnetic moments in the region between magnetic domains may be temperature independent when averaged over the scale of the mean free path of conduction electrons. As an alternative, it may be that the coherent and incoherent electron-magnon scattering mechanisms cancel quantitatively when summed, thus giving a temperature-independent resistivity at low temperatures. ${ }^{33}$

With regard to the correlation between the unidirectional exchange anisotropy and coercivity and the resistance minimum (parameters are summarized in Tables I and II), the larger the unidirectional exchange anisotropy the more pronounced is the minimum that occurs. Since the Dzyaloshinski-Moriya (DM) and uniaxial anisotropy effects lead to irreversibility in RSG systems, ${ }^{34}$ one can assert that an exchange anisotropy including both uniaxial and DM anisotropic interactions plays an important role in the temperature dependence of the resistivity. Indeed, the $\mathrm{Ni}_{74} \mathrm{Mn}_{24} \mathrm{Pd}_{2}$ sample shows a much deeper minimum at higher temperature. The irreversibility effect in this sample is much more severe due to anisotropic exchange interactions within the domains and on the domain surfaces. This sample takes place just on the border of the RSG such that it could be considered a superparamagnetic sample. Thus this correlation provides us a separate measure of the presence of antiferromagnetically coupled domains in these materials.

We conclude that in these reentrant spin-glass (RSG) systems, anisotropic exchange interactions manifest themselves through the resistivity, giving rise to an additional term as described here. The temperature behavior of this contribution can be explained satisfactorily in terms of magnetic two-level-type interactions mediated by electron-spindependent scattering between two channels. The temperature-dependent resistivity measurements show that a Kondo-like logarithmic temperature characteristic changes to a power-law behavior going as $T^{1 / 2}$ as the temperature is further decreased. Fitting parameters such as the resistivity coefficients are closely correlated with the irreversibility effects characterized by coercivities and the magnetic freezing temperatures. Thus, the anomalous behavior observed in the resistivity can be attributed to the irreversibility effects for RSG systems.

\section{ACKNOWLEDGMENTS}

This work was supported by the Robert A. Welch Foundation, Grant No. A-1526. One of us (Y.Ö.) would also like to thank for the support of the Istanbul Technical University Research Fund (Project No. 855).

${ }^{1}$ W. Abdul-Razzaq and J. S. Kouvel, Phys. Rev. B 35, 1764 (1987)

${ }^{2}$ T. Sato, T. Ando, T. Ogawa, S. Morimoto, and A. Ito, Phys. Rev. B 64, $184432(2001)$

${ }^{3}$ S. F. Fischer, S. N. Kaul, and H. Kronmüller, Phys. Rev. B 65, 064443 (2002).

${ }^{4}$ A. Janutka, J. Phys.: Condens. Matter 15, 8561 (2003).

${ }^{5}$ W. Bao, S. Raymond, S. M. Shapiro, K. Motoya, B. Fåk, and R. W. Erwin, Phys. Rev. Lett. 82, 4711 (1999).

${ }^{6}$ S. Senoussi and Y. Oner, Phys. Rev. B 28, R455 (1983).

${ }^{7}$ Y. Oner, A. Kılıc, and H. Celik, Physica B 215, 205 (1995).

${ }^{8}$ T. Sato, N. Yoneyama, Y. Torii, and T. Ando, J. Magn. Magn. Mater. 90, 349 (1990).

${ }^{9}$ M. Olivier, J. O. Strom-Olsen, and Z. Altounian, Phys. Rev. B 35, 333 (1987), and references therein.

${ }^{10}$ R. W. Cochrane, R. Harris, J. O. Strom-Olsen, and M. J. Zuckerman, Phys. Rev. Lett. 35, 676 (1975).

${ }^{11}$ M. A. Howson and B. L. Gallagher, Phys. Rep. 170, 265 (1988).

${ }^{12}$ B. Aktas, Y. Öner, and H. Z. Durusoy, J. Magn. Magn. Mater. 119, 339 (1993).

${ }^{13}$ Y. Öner, C. S. Lue, J. H. Ross, Jr., and K. D. D. Rathnayaka, J. Appl. Phys. 89, 7044 (2001).

${ }^{14}$ J. M. Ziman, Principles of the Theory of Solids (Cambridge University Press, London, 1979).

${ }^{15}$ Y. P. Lee, K. W. Kim, J. Y. Rhee, Y. V. Kudryavtsev, V. V. Nemoshkalenko, and V. G. Prokhorov, Eur. Phys. J. B 15, 451 (2000).

${ }^{16}$ A. Berrada, M. F. Lapierre, B. Loegel, P. Panissod, and C. Robert, J. Phys. F: Met. Phys. 8, 845 (1978).

${ }^{17}$ Y. Yamada, Y. Itoh, U. Mizutani, N. Shibagaki, and K. Tanaka, J. Phys. F: Met. Phys. 17, 2303 (1987).

${ }^{18}$ R. W. Cochrane and J. O. Strom-Olsen, Phys. Rev. B 29, 1088 (1984).

${ }^{19}$ H. Z. Durusoy and Y. Oner, Phys. Rev. B 42, R 6831 (1990).

${ }^{20}$ E. M. Gyorgy, L. R. Walker, and J. H. Wernick, Phys. Rev. Lett. 51, 1684 (1983).

${ }^{21}$ I. Ya. Korenblit, S. V. Maleev, and E. F. Shender, Phys. Rev. B 33, 624 (1986).

${ }^{22}$ G. Aeppli, S. M. Shapiro, R. J. Birgeneau, and H. S. Chen, Phys. Rev. B 29, 2589 (1984).

${ }^{23}$ M. A. Continentino, Phys. Rev. B 27, 4351 (1983).

${ }^{24}$ Y. Öner, M. Özdemir, B. Aktas, C. Topacli, E. A. Harris, and S. Senoussi, J. Magn. Magn. Mater. 170, 129 (1997).

${ }^{25}$ D. M. Herlach, E. F. Wassermann, and R. Willnecker, Phys. Rev. Lett. 50, 529 (1983).

${ }^{26}$ E. F. Wassermann and D. M. Herlach, J. Appl. Phys. 55, 1709 (1984).

${ }^{27}$ D. L. Cox and A. Zawadowski, Adv. Phys. 47, 599 (1998), and references therein.

${ }^{28}$ T. Cichorek, H. Aoki, J. Custers, P. Gegenwart, F. Steglich, Z. Henkie, E. D. Bauer, and M. B. Maple, Phys. Rev. B 68, 144411 (2003).

${ }^{29}$ T. Cichorek, Z. Henkie, J. Custers, P. Gegenwart, and F. Steglich, J. Magn. Magn. Mater. 66-67, 272 (2004).

${ }^{30}$ I. L. Aleiner, B. L. Altshuler, Y. M. Galperin, and T. A. Shutenko, Phys. Rev. Lett. 86, 2629 (2001).

${ }^{31}$ G. Zaránd, Phys. Rev. B 72, 245103 (2005).

${ }^{32}$ A. Fert and P. M. Levy, Phys. Rev. Lett. 44, 1538 (1980).

${ }^{33}$ G. Bergmann and P. Marquardt, Phys. Rev. B 17, 1355 (1978).

${ }^{34}$ I. A. Campbell, H. Hurdequint, and F. Hippert, Phys. Rev. B 33, 3540 (1986). 